\title{
Construction of Graeco Sudoku Square Designs of Odd Orders
}

\section{J. Subramani}

\begin{abstract}
The Sudoku puzzle typically consists of a nineby-nine grid, in which some of the spaces contain numbers; most of the spaces are blank. The goal is to fill in the blanks with digits from 1 to 9 so that each row, each column, and each of the nine three-by-three blocks making up the grid contains just one of each of the nine digits. Recently Subramani and Ponnuswamy (2009) have considered the Sudoku puzzle as an experimental design and introduced the concept of Sudoku designs. The Sudoku designs are similar to that of latin square designs but accommodate some additional factors. The method of constructing the Sudoku square designs, analysis and applications are also given by Subramani and Ponnuswamy (2009). In this paper we have extended the Sudoku designs to Orthogonal (Graeco) Sudoku square designs in line with that of the Orthogonal (Graeco) latin square designs. A simple method of constructing Graeco Sudoku square designs (GSSD) of odd orders is presented. The proposed method is explained with the help of numerical examples.
\end{abstract}

Keywords--- Construction, Latin Squares, Orthogonal Latin Squares, Sudoku Square Designs, Sequential Method

\section{INTRODUCTION}

$\mathrm{I}_{\mathrm{T}}^{\mathrm{T}}$ $\mathrm{T}$ is not execration to say that millions of people in Japan, Great Britain, and elsewhere around the world are addict in making an attempt to solve a simple number puzzle in every day morning and are finding that a day isn't complete without tackling the latest edition of a simple puzzle called sudoku. The Sudoku puzzle typically consists of a nine-by-nine grid in which some of the spaces contain numbers; most of the spaces are blank. The purpose of the puzzle or goal is to fill in the blanks with digits from 1 to 9 so that each row, each column, and each of the nine three-by-three blocks contains the numbers from 1 to 9 only once. Even though the rules are very simple, the puzzles are very challenging and also highly addictive. The name "Sudoku" is the abbreviation of the Japanese longer phrase, "Sūji wa dokushin ni kagiru",

which means "the digits must occur only once". It is a trademark of puzzle publisher Nikoli Co. Ltd. in Japan. The sudoku puzzles are referred by other Japanese publishers as Number Place, the original U.S. title, or as "Nanpure" for short. Some non-Japanese publishers spell the title as "Su 'Doku".

J. Subramani, Associate Professor, Department of Statistics, Pondicherry University, Puducherry, India. E-mail: drjsubramani@yahoo.co.in
The sudoku puzzles can also be decorated by using any other symbols other than the numerals such that every symbol will occur only once in every row, column and subsquares. For example the numerals can be replaced by alphabets or letters of any language, shapes, or colours. Dell Magazines, the puzzle's originator, has been using numerals for Number Place in its magazines since they first published it in 1979. It is to be noted that the sudoku puzzle with $9 \times 9$ grid with $3 \times 3$ regions is by far the most common, variations abound: sample puzzles can be $4 \times 4$ grids with $2 \times 2$ regions; $5 \times 5$ grids with pentomino regions; $6 \times 6$ grids with $2 \times 3$ regions and a $7 \times 7$ grid with six heptomino regions and a disjoint region. Larger grids are also possible, with Daily SuDoku's 16×16-grid Monster SuDoku, the Times likewise offers a $12 \times 12$-grid Dodeka sudoku with 12 regions each being $4 \times 3$, Dell regularly publishing $16 \times 16$ Number Place Challenger puzzles and Nikoli proffering $25 \times 25$ Sudoku the Giant behemoths. Even larger sizes, for instance $100 \times 100$, have been claimed.Sudoku puzzle is basically a logic puzzle and there is no mathematics involved in solving the puzzles. The digits from 1 to 9 can be easily replaced by 9 different alphabets or letters of any language, shapes, colours or even by names. There is mathematics and computer science, however, in analyzing the puzzles and creating efficient computer programs for generating and solving them.

Solving a sudoku puzzles can be extremely difficult depending on the number of clues and the size of the grid. It is proved by Takayuki Yato and Takahiro Seta [6] of the University of Tokyo that the problem of solving $n$-by- $n$ sudoku puzzles in general belongs to a category of computational problems described as NP-complete. An NP problem is one for which it's relatively easy to check whether a given answer is correct but may require an impossibly long time to solve by any direct procedure, especially as $n$ gets larger and larger. Indeed, as the number of elements, $n$, increases, a computer's solution time grows exponentially in the worst case.

A sudoku grid is closely connected with the mathematical object called a Latin square. A Latin square of order $n$ is an arrangement of $n$ numbers from 1 to $n$ arranged in a square array (matrix) so that every row or every column contains all the $n$ numbers only once. There are two Latin squares of order two $(n=2), 12$ of order three, 576 of order four and 5524751496156892842531225600 of order 9. Latin squares go back to at least medieval times. Leonhard Euler (17071783) was the first mathematician to study them systematically. He introduced a particular type of Latin square (a Graeco-Roman square) as a new kind of "magic square." As in sudoku, the rows and columns of a Latin square don't have to be filled with numbers. Any set of $n$ different symbols will 
do. The additional constraint that a standard nine-by-nine sudoku puzzle has three-by-three blocks that also contain each of the nine digits reduces the enormous number of possible nine-by-nine Latin squares to a smaller but still-humungous number: 6670903752021072936960. The readers are referred to see http://www.sudoku.com/forums/viewtopic.php?t=44\&start=13 8 for a discussion on how Bertram Felgenhauer of Dresden, Germany, obtained this number, which represents how many unique, one-solution puzzles can be produced. Given all those possibilities, there'll certainly be no shortage of material to feed sudoku addiction. For various details on Sudoku puzzles one may refer the websites given in [4]-[10].

The problem of constructing orthogonal Latin squares has drawn the attention of many leading mathematicians and statisticians for a longer period. Das and Dey [1] have presented a sequential method of constructing a pair of orthogonal Latin squares of odd orders by developing two initial rows. By super imposing any two orthogonal Latin squares one may get a Graeco Latin square. That is, the problem of constructing a Graeco Latin square and a pair of orthogonal Latin squares are one and the same. Subramani [2] has extended the method of Das and Dey [1] and introduced a more generalized method for constructing several orthogonal Latin squares of odd orders for a given Latin square. In this connection, Subramani [2] has presented the Theorem 1, which ensures the existence of a pair of orthogonal Latin squares of odd orders.

Let $m^{2}(=2 n+1)$ be the number of symbols coded by $\left(1,2,3, \ldots, m^{2}\right)$ and is denoted by the set $S=\left(1,2,3, \ldots, m^{2}\right)$. A pair of orthogonal Latin squares is obtained by generating the two initial rows. It is to be noted that throughout this paper $m^{2}$ represents an odd number, consequently $m$ is also an odd number unless otherwise stated explicitly.

Theorem 1: A Graeco Latin square (equivalently, a pair of orthogonal Latin squares) of odd order $m^{2}$ can be obtained by developing the following two initial rows.

$$
\begin{aligned}
& A_{1}=\left\{i_{1}, i_{2}, i_{3}, \ldots, i_{k}, \ldots, i_{m^{2}}\right\} \bmod m^{2} \\
& B_{1}=\left\{j_{1}, j_{2}, j_{3}, \ldots, j_{k}, \ldots, j_{m^{2}}\right\} \bmod m^{2} \\
& \text { provided (i) } i_{k} \neq i_{l} \text { if } k \neq l \\
& \text { (ii) } j_{k} \neq j_{l} \text { if } k \neq l \\
& \text { (iii) } i_{k}+j_{k}=p, \quad \mathrm{k}=1,2,3, \ldots, m^{2}
\end{aligned}
$$

where $i_{k}, j_{k}$ and $p$ are the members of the set $\mathrm{S}=\left(1,2,3, \ldots, \mathrm{m}^{2}\right)$.

For the proof of the above theorem and for other details the readers are referred to Subramani [2].

Recently Subramani and Ponnuswamy [3] have introduced a new experimental design namely Sudoku design based on the Sudoku puzzles. They have also provided the construction, analysis and applications of Sudoku designs and explained them with the help of numerical examples. For further details on the definition, design, analysis and applications of Sudoku square designs one may refer to Subramani and Ponnuswamy [3] and the references cited therein.

In this paper an attempt is made to extend the method of Subramani [2], the construction of orthogonal Latin square designs of odd orders to construct orthogonal Sudoku square designs (or equivalently Graeco Sudoku square design) of odd orders. The sequential method of construction of orthogonal Sudoku square designs is presented in section 2 together with some other related results and numerical examples.

\section{Construction of ORTHOgOnal SUdOKU SQUARE DESIGNS}

Definition 1: A Sudoku square consists of $m^{2}$ sets of numbers from 1 to $\mathrm{m}^{2}$ in a square array so that no row or column contains the same number more than once together with the additional constraint that each of the $\mathrm{m}^{2}$ sub squares of order $m \times m$ contains the set of $m^{2}$ numbers only once. That is, a Sudoku square is a special case of a Latin square.

Definition 2: Two Sudoku squares of order $\mathrm{m}^{2}$ are said to be mutually orthogonal Sudoku squares, if we super impose the two Sudoku squares then one may get each pair of numbers only once.

To construct Sudoku designs of order $\mathrm{m}^{2}$ sequentially, follow the steps given below:

1. Write the $m^{2}$ numbers from 1 to $m^{2}$ in a matrix form sequentially starting from column 1 to column $\mathrm{m}$ (as given in Fig.1.)

2. Write the $m$ rows obtained in step 1 , side by side to get a row of order $m^{2}$ as given in equation (2).

3. Row 2 can be obtained from row 1 by adding 1 to each of its elements and reduce to mod $m^{2}$ if it exceeds the value $m^{2}$. Proceed in the similar way to complete all the $m^{2}$ rows.

Alternatively one may generate a Sudoku square by developing the initial row sequentially

$$
\begin{aligned}
& \{1, m+1, \ldots,(m-1) m+1,2, m+2, \ldots,(m-1) m+2, \ldots, m, \\
& \left.2 m, \ldots,(m-1) m+m=m^{2}\right\}
\end{aligned}
$$

It is well known that if the initial (first) row contains all the elements from 1 to $\mathrm{m}^{2}$ only once then it will generate a Latin square due to the fact that by adding a constant to each of the numbers and reduce to mod $\mathrm{m}^{2}$ one may get the same set but in different orders. Here it is enough to prove that each of the numbers in the set appears only once in every sub squares. This can be easily proved from the following. Each of the rows of the Fig. 1 generates the square of order $m$ with all the elements from 1 to $\mathrm{m}^{2}$ appear once. 


\begin{tabular}{|c|c|c|c|c|c|}
\hline \multicolumn{2}{|c|}{} & \multicolumn{5}{|c|}{ Columns } \\
\cline { 3 - 6 } \multicolumn{2}{|c|}{} & 1 & 2 & $\ldots$ & $m$ \\
\hline \multirow{4}{*}{ Rows } & 1 & 1 & $m+1$ & $\ldots$ & $(m-1) m$ \\
\cline { 2 - 6 } & 2 & 2 & $m+2$ & & $(m-1) m$ \\
\cline { 2 - 6 } & $\vdots$ & $\vdots$ & $\vdots$ & $\vdots$ & $\vdots$ \\
\cline { 2 - 6 } & $m$ & $m$ & $2 m$ & & $m^{2}$ \\
\hline
\end{tabular}

Figure 1: Generates a Sub Square of a Sudoku Square
For example, the first row in Fig. 1 has generated a matrix of order $m$. Now, by counting the numbers from column 1 to column $m$ one may see all the numbers from 1 to $\mathrm{m}^{2}$ appear only once. Further if we add any integer to the numbers from 1 and $m^{2}$, and reduce to $\bmod m^{2}$ we may get the same set but in different orders. The method given above can be explained with the help of the example as given below:

\begin{tabular}{|c|c|c|c|c|c|c|c|c|c|c|c|c|c|}
\hline & 1 & 2 & $\ldots$ & $\mathrm{m}$ & $\mathrm{m}+1$ & $\mathrm{~m}+2$ & $\ldots$ & $2 \mathrm{~m}$ & $\cdots$ & $\begin{array}{c}(\mathrm{m}-1) \\
\mathrm{m}+1\end{array}$ & $\begin{array}{c}(\mathrm{m}-1) \\
\mathrm{m}+2\end{array}$ & $\cdots$ & $\mathrm{m} 2$ \\
\hline 1 & 1 & 2 & $\ldots$ & $\mathrm{m}$ & $\mathrm{m}+1$ & $\mathrm{~m}+2$ & $\ldots$ & $2 \mathrm{~m}$ & $\ldots$ & $\begin{array}{c}(\mathrm{m}-1) \\
\mathrm{m}+1\end{array}$ & $\begin{array}{c}\mathrm{m}-1) \\
\mathrm{m}+2\end{array}$ & $\cdots$ & $\mathrm{m} 2$ \\
\hline 2 & $\mathrm{~m}+1$ & $\mathrm{~m}+2$ & $\ldots$ & $2 \mathrm{~m}$ & $2 m+1$ & $2 m+2$ & $\ldots$ & $3 \mathrm{~m}$ & $\ldots$ & 1 & 2 & $\ldots$ & $\mathrm{m}$ \\
\hline . & . & . & $\cdots$ & . & . & . & $\cdots$ & . & $\cdots$ & . & . & $\cdots$ & . \\
\hline $\mathrm{m}$ & $\begin{array}{c}(\mathrm{m}-1) \\
\mathrm{m}+1\end{array}$ & $\begin{array}{c}(\mathrm{m}-1) \\
\mathrm{m}+2\end{array}$ & $\cdots$ & $\mathrm{m} 2$ & 1 & 2 & $\cdots$ & $\mathrm{m}$ & $\cdots$ & $\begin{array}{c}(\mathrm{m}-2) \\
\mathrm{m}+1\end{array}$ & $\begin{array}{c}(\mathrm{m}-2) \\
\mathrm{m}+2\end{array}$ & $\cdots$ & $\begin{array}{c}(\mathrm{m}-1) \\
\mathrm{m}\end{array}$ \\
\hline $\mathrm{m}+1$ & 2 & 3 & $\ldots$ & $\mathrm{m}+1$ & $\mathrm{~m}+2$ & $\mathrm{~m}+3$ & $\ldots$ & $2 m+1$ & $\cdots$ & $\begin{array}{c}(\mathrm{m}-1) \\
\mathrm{m}+2\end{array}$ & $\begin{array}{c}\mathrm{m}-1) \\
\mathrm{m}+3\end{array}$ & $\ldots$ & 1 \\
\hline $\mathrm{m}+2$ & $\mathrm{~m}+2$ & $\mathrm{~m}+3$ & $\ldots$ & $2 m+1$ & $2 m+2$ & $2 m+3$ & $\ldots$ & $3 m+1$ & $\ldots$ & 2 & 3 & $\ldots$ & $\mathrm{m}+1$ \\
\hline . & . & . & $\ldots$ & . & . & . & $\cdots$ & . & $\cdots$ & . & . & $\cdots$ & . \\
\hline $2 \mathrm{~m}$ & $\begin{array}{c}\mathrm{m}-1) \\
\mathrm{m}+2\end{array}$ & $\begin{array}{c}(\mathrm{m}-1) \\
\mathrm{m}+3\end{array}$ & $\cdots$ & 1 & 2 & 3 & $\ldots$ & $\mathrm{m}+1$ & $\cdots$ & $\begin{array}{c}(\mathrm{m}-2) \\
\mathrm{m}+2\end{array}$ & $\begin{array}{c}\mathrm{m}-2) \\
\mathrm{m}+3\end{array}$ & $\cdots$ & $\begin{array}{c}(\mathrm{m}-2) \\
\mathrm{m}+1\end{array}$ \\
\hline . & . & . & $\ldots$ & . & . & . & $\cdots$ & . & $\cdots$ & . & $\cdot$ & $\cdots$ & $\cdot$ \\
\hline $\begin{array}{c}(\mathrm{m}-1) \\
\mathrm{m}+1\end{array}$ & $\mathrm{~m}$ & $\mathrm{~m}+1$ & $\cdots$ & $2 \mathrm{~m}-1$ & $2 \mathrm{~m}$ & $2 m+1$ & $\cdots$ & $3 m-1$ & $\cdots$ & $\mathrm{m} 2$ & 1 & $\cdots$ & $\mathrm{m}-1$ \\
\hline $\begin{array}{c}(\mathrm{m}-1) \\
\mathrm{m}+2\end{array}$ & $2 \mathrm{~m}$ & $2 \mathrm{~m}+1$ & $\ldots$ & $3 m-1$ & $3 \mathrm{~m}$ & $3 m+1$ & $\ldots$ & $4 m-1$ & $\ldots$ & $\mathrm{m}$ & $\mathrm{m}+1$ & $\ldots$ & $2 \mathrm{~m}-1$ \\
\hline . & . & . & $\ldots$ & . & . & . & $\cdots$ & . & $\cdots$ & . & . & $\cdots$ & . \\
\hline $\mathrm{m} 2$ & $\mathrm{~m} 2$ & 1 & $\ldots$ & $\mathrm{m}-1$ & $\mathrm{~m}$ & $\mathrm{~m}+1$ & $\ldots$ & $2 \mathrm{~m}-1$ & $\cdots$ & $\begin{array}{c}(\mathrm{m}-1) \\
\mathrm{m}\end{array}$ & $\begin{array}{c}(\mathrm{m}-1) \\
\mathrm{m}+1\end{array}$ & $\cdots$ & m-1 \\
\hline
\end{tabular}

Figure 2: Sudoku Square of Order $\mathrm{m}^{2}$

\begin{tabular}{|l|l|l|l|l|l|l|l|l|}
\hline $\mathbf{1}$ & $\mathbf{4}$ & $\mathbf{7}$ & $\mathbf{2}$ & $\mathbf{5}$ & $\mathbf{8}$ & $\mathbf{3}$ & $\mathbf{6}$ & $\mathbf{9}$ \\
\hline 2 & 5 & 8 & 3 & 6 & 9 & 4 & 7 & 1 \\
\hline 3 & 6 & 9 & 4 & 7 & 1 & 5 & 8 & 2 \\
\hline $\mathbf{4}$ & $\mathbf{7}$ & $\mathbf{1}$ & $\mathbf{5}$ & $\mathbf{8}$ & $\mathbf{2}$ & $\mathbf{6}$ & $\mathbf{9}$ & $\mathbf{3}$ \\
\hline 5 & 8 & 2 & 6 & 9 & 3 & 7 & 1 & 4 \\
\hline 6 & 9 & 3 & 7 & 1 & 4 & 8 & 2 & 5 \\
\hline $\mathbf{7}$ & $\mathbf{1}$ & $\mathbf{4}$ & $\mathbf{8}$ & $\mathbf{2}$ & $\mathbf{5}$ & $\mathbf{9}$ & $\mathbf{3}$ & $\mathbf{6}$ \\
\hline 8 & 2 & 5 & 9 & 3 & 6 & 1 & 4 & 7 \\
\hline 9 & 3 & 6 & 1 & 4 & 7 & 2 & 5 & 8 \\
\hline
\end{tabular}

Figure 3: Sudoku Square of Order 9

It is to be noted that the numbers in the first row of each of the sub squares generates the matrix of order 3 with numbers 1 to 9 appears only once.

The following example explains the method of constructing an orthogonal Sudoku square of order 9 for the given Sudoku square (Fig. 3). Let the first row of the Sudoku square is $\{1,4,7,2,5,8,3,6,9\}$. Then the first row of its orthogonal
Sudoku square for $p=1$ is $\{9,6,3,8,5,2,7,4,1\}$ and the corresponding Sudoku square is given below: 


\begin{tabular}{|l|l|l|l|l|l|l|l|l|}
\hline 9 & 6 & 3 & 8 & 5 & 2 & 7 & 4 & 1 \\
\hline 1 & 7 & 4 & 9 & 6 & 3 & 8 & 5 & 2 \\
\hline 2 & 8 & 5 & 1 & 7 & 4 & 9 & 6 & 3 \\
\hline 3 & 9 & 6 & 2 & 8 & 5 & 1 & 7 & 4 \\
\hline 4 & 1 & 7 & 3 & 9 & 6 & 2 & 8 & 5 \\
\hline 5 & 2 & 8 & 4 & 1 & 7 & 3 & 9 & 6 \\
\hline 6 & 3 & 9 & 5 & 2 & 8 & 4 & 1 & 7 \\
\hline 7 & 4 & 1 & 6 & 3 & 9 & 5 & 2 & 8 \\
\hline 8 & 5 & 2 & 7 & 4 & 1 & 6 & 3 & 9 \\
\hline
\end{tabular}

Figure 4: Sudoku Square Orthogonal to Fig. 3

By super imposing the two Sudoku squares given in Fig. 3 and Fig. 4 one may get the orthogonal (Graeco) Sudoku squares given in Fig.5.

\begin{tabular}{|l|l|l|l|l|l|l|l|l|}
\hline$(1,9)$ & $(4,6)$ & $(7,3)$ & $(2,8)$ & $(5,5)$ & $(8,2)$ & $(3,7)$ & $(6,4)$ & $(9,1)$ \\
\hline$(2,1)$ & $(5,7)$ & $(8,4)$ & $(3,9)$ & $(6,6)$ & $(9,3)$ & $(4,8)$ & $(7,5)$ & $(1,2)$ \\
\hline$(3,2)$ & $(6,8)$ & $(9,5)$ & $(4,1)$ & $(7,7)$ & $(1,4)$ & $(5,9)$ & $(8,6)$ & $(2,3)$ \\
\hline$(4,3)$ & $(7,9)$ & $(1,6)$ & $(5,2)$ & $(8,8)$ & $(2,5)$ & $(6,1)$ & $(9,7)$ & $(3,4)$ \\
\hline$(5,4)$ & $(8,1)$ & $(2,7)$ & $(6,3)$ & $(9,9)$ & $(3,6)$ & $(7,2)$ & $(1,8)$ & $(4,5)$ \\
\hline$(6,5)$ & $(9,2)$ & $(3,8)$ & $(7,4)$ & $(1,1)$ & $(4,7)$ & $(8,3)$ & $(2,9)$ & $(5,6)$ \\
\hline$(7,6)$ & $(1,3)$ & $(4,9)$ & $(8,5)$ & $(2,2)$ & $(5,8)$ & $(9,4)$ & $(3,1)$ & $(6,7)$ \\
\hline$(8,7)$ & $(2,4)$ & $(5,1)$ & $(9,6)$ & $(3,3)$ & $(6,9)$ & $(1,5)$ & $(4,2)$ & $(7,8)$ \\
\hline$(9,8)$ & $(3,5)$ & $(6,2)$ & $(1,7)$ & $(4,4)$ & $(7,1)$ & $(2,6)$ & $(5,3)$ & $(8,9)$ \\
\hline
\end{tabular}

Figure 5: A Pair of Orthogonal Sudoku Squares when $p_{=1}$

Remark 1: It is to be noted that the proposed method of constructing Sudoku designs is valid for any value of $m^{2}$ (odd or even) whereas the construction of orthogonal Sudoku square designs is valid for odd value of $m^{2}$.

If $p=2$, then the two initial rows $\{1,4,7,2,5,8,3,6,9\}$ and $\{1,7,4,9,6,3,8,5,2\}$ generates the orthogonal Sudoku squares as given in Fig. 6.

\begin{tabular}{|c|c|c|c|c|c|c|c|c|}
\hline$(1,1)$ & $(4,7)$ & $(7,4)$ & $(2,9)$ & $(5,6)$ & $(8,3)$ & $(3,8)$ & $(6,5)$ & $(9,2)$ \\
\hline$(2,2)$ & $(5,8)$ & $(8,5)$ & $(3,1)$ & $(6,7)$ & $(9,4)$ & $(4,9)$ & $(7,6)$ & $(1,3)$ \\
\hline$(3,3)$ & $(6,9)$ & $(9,6)$ & $(4,2)$ & $(7,8)$ & $(1,5)$ & $(5,1)$ & $(8,7)$ & $(2,4)$ \\
\hline$(4,4)$ & $(7,1)$ & $(1,7)$ & $(5,3)$ & $(8,9)$ & $(2,6)$ & $(6,2)$ & $(9,8)$ & $(3,5)$ \\
\hline$(5,5)$ & $(8,2)$ & $(2,8)$ & $(6,4)$ & $(9,1)$ & $(3,7)$ & $(7,3)$ & $(1,9)$ & $(4,6)$ \\
\hline$(6,6)$ & $(9,3)$ & $(3,9)$ & $(7,5)$ & $(1,2)$ & $(4,8)$ & $(8,4)$ & $(2,1)$ & $(5,7)$ \\
\hline$(7,7)$ & $(1,4)$ & $(4,1)$ & $(8,6)$ & $(2,3)$ & $(5,9)$ & $(9,5)$ & $(3,2)$ & $(6,8)$ \\
\hline$(8,8)$ & $(2,5)$ & $(5,2)$ & $(9,7)$ & $(3,4)$ & $(6,1)$ & $(1,6)$ & $(4,3)$ & $(7,9)$ \\
\hline$(9,9)$ & $(3,6)$ & $(6,3)$ & $(1,8)$ & $(4,5)$ & $(7,2)$ & $(2,7)$ & $(5,4)$ & $(8,1)$ \\
\hline
\end{tabular}

Fig. 6: A pair of orthogonal Sudoku squares when $p=2$

In the similar way by taking different values for $p$ from 1 to $m^{2}$ one may construct different orthogonal Sudoku squares for the given Sudoku square. For example, let $A_{1}$ and $B_{1}$ be the first (initial) rows of the orthogonal Sudoku squares, where $A_{1}=\left\{i_{1}, i_{2}, i_{3}, \ldots, i_{k}, \ldots, i_{m^{2}}\right\}$ and $\quad B_{1}=\left\{j_{1}, j_{2}, j_{3}, \ldots, j_{k}, \ldots, j_{m^{2}}\right\}$. When $m=3$, for the given $A_{1}=\{1,4,7,2,5,8,3,6,9\}$ one may get 9 different choices for $B_{1}=\left\{j_{1}, j_{2}, j_{3}, \ldots, j_{k}, \ldots, j_{m^{2}}\right\}$ for different values of $p$ from 1 to 9 as given below:

$$
\begin{array}{ll}
B_{1}=\{9,6,3,8,5,2,7,4,1\} & \text { for } p=1 \\
B_{1}=\{1,7,4,9,6,3,8,5,2\} & \text { for } p=2
\end{array}
$$

$$
\begin{array}{ll}
B_{1}=\{2,8,5,1,7,4,9,6,3\} & \text { for } p=3 \\
B_{1}=\{3,9,6,2,8,5,1,7,4\} & \text { for } p=4 \\
B_{1}=\{4,1,7,3,9,6,2,8,5\} & \text { for } p=5 \\
B_{1}=\{5,2,8,4,1,8,3,9,6\} & \text { for } p=6 \\
B_{1}=\{6,3,9,5,2,8,4,1,7\} & \text { for } p=7 \\
B_{1}=\{7,4,1,6,3,9,5,2,8\} & \text { for } p=8 \\
B_{1}=\{8,5,2,7,4,1,6,3,9\} & \text { for } p=9
\end{array}
$$

That is, for the given Sudoku square one may easily construct $m^{2}$ different orthogonal Sudoku squares.

From the above discussions we arrive at the following theorems.

Theorem 2: A Graeco Sudoku squares of order $m^{2}(=2 n+1)$ can be constructed by developing the following two initial rows.

$$
\begin{aligned}
& A_{1}=\left\{i_{1}, i_{2}, i_{3}, \ldots, i_{k}, \ldots, i_{m^{2}}\right\} \bmod m^{2} \\
& =\{1, m+1, \ldots,(m-1) m+1,2, m+2, \ldots,(m-1) m+2, \ldots, m, \\
& \left.2 m, \ldots,(m-1) m+m=m^{2}\right\} \bmod m^{2} \\
& B_{1}=\left\{j_{1}, j_{2}, j_{3}, \ldots, j_{k}, \ldots, j_{m^{2}}\right\} \bmod m^{2}
\end{aligned}
$$

provided (i) $i_{k} \neq i_{l}$ if $k \neq l$

$$
\begin{aligned}
& \text { (ii) } j_{k} \neq j_{l} \text { if } k \neq l \\
& \text { (iii) } i_{k}+j_{k}=p, \quad \mathrm{k}=1,2,3, \ldots, m^{2}
\end{aligned}
$$

where $i_{k}, j_{k}$ and $p$ are the members of the set $\mathrm{S}=\left(1,2,3, \ldots, m^{2}\right)$.

The proof is similar to that of Theorem 1 and hence it is omitted.

Theorem 3: From a given Sudoku square of order $m^{2}$, one can always obtain $m^{2}$ distinct Graeco Sudoku squares.

Proof: Let $i_{k}$ be the $\mathrm{k}^{\text {th }}$ element of the initial block of the given Sudoku square. From Theorem 2 one can easily obtain the Graeco Sudoku square with $\mathrm{k}^{\text {th }}$ element of the initial block as $\left(i_{k}, j_{k}\right)$ such that $i_{k}+j_{k} \bmod m^{2}=p$, where $p$ takes any value in the set $\mathrm{S}=\left\{1,2,3, \ldots, \mathrm{m}^{2}\right\}$. Hence the proof.

Theorem 4: There always exist $(m !)^{2(m+1)} \times m^{2}$ distinct Graeco Sudoku squares of order $m^{2}$

Proof: Let $\left(\mathrm{i}_{1}, \mathrm{i}_{2}, \ldots, \mathrm{i}_{\mathrm{m}^{2}}\right)$ be the initial row of a Sudoku square of order $m^{2}$. By the permutation of the sub squares and also the numbers within each of the sub squares in the initial row one can obtain $(m !)^{m+1}$ distinct initial rows which lead to $(m !)^{m+1}$ distinct Sudoku squares of order $m^{2}$. By applying Theorem 3 we can always construct $(m !)^{m+1} \times m^{2}$ Graeco 
Sudoku squares. Further by the permutation of the sub squares and also the rows within each of the sub squares one can obtain $(m !)^{m+1}$ distinct Graeco Sudoku squares which lead to $(m !)^{m+1} \times(m !)^{m+1} \times m^{2}=(m !)^{2(m+1)} \times m^{2}$ distinct Graeco Sudoku squares of order $m^{2}$.

\section{Example}

It is given earlier that the initial row $A_{1}=\{1,4,7,2,5,8,3,6,9\} \bmod 9$ can generate a Sudoku square of order 9. Further we know that by interchanging any two numbers within the sub squares (alternatively any two columns within the sub squares) will not alter the property of the Sudoku designs, which leads to $(m !)^{2(m+1)} \times m^{2}=(3 !)^{2(3+1)} \times 3^{2}=6^{8} \times 9=15116544 \quad$ Graeco

Sudoku squares of order 9 .

\section{REFERENCE}

[1] A. Das and A. Dey, "A Note on Construction of Graeco Latin Square of order 2n+1”, J. Indian Soc. Agric. Statist., 42, 247-249, 1990.

[2] J. Subramani. A Further Note on Construction of Graeco Latin Square of order 2n+1. J. Indian Soc. Agric. Statist., 48, 356-359,1996

[3] J. Subramani and K.N.Ponnuswamy. Construction and analysis of Sudoku designs, Model Assisted Statistics and Applications 4, 287-301, 2009

[4] I. Peterson, Proof by computer. Science News Online (Oct. 2). Available at http://www.sciencenews.org/articles/20041002/mathtrek.asp., 2004

[5] Completing Latin squares. Science News Online (May 6). Available at http://www.sciencenews.org/articles/20000506/mathtrek.asp, 2000

[6] T.Yato, and T. Seta. Preprint. Complexity and completeness of finding another solution and its application to puzzles. Available at http://www.phil.uu.nl/ oostrom/cki20/02-03/japansepuzzles/ASP.pdf.

[7] Information about sudoku can be found at Wayne Gould's Web site at http://www.sudoku.com/ and http://en.wikipedia.org/wiki/Sudoku.

[8] The London Times sudoko page is at http://www.timesonline.co.uk/section/0,,18209,00.html.

[9] Online sudoku puzzles are available at http://www.websudoku.com/, http://www.fingertime.com/sudoku.php, http://www.sudoku.name/playsudoku.php?ln=en, and http://www.dailysudoku.co.uk/sudoku/.

[10] A sudoku solver is available at http://www.sudoku-solver.com/ and http://sudoku.sourceforge.net/.

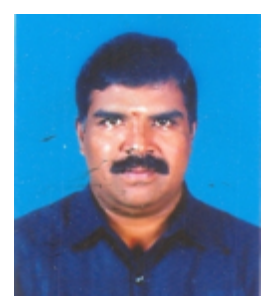

Dr. J. Subramani has received the doctorate degree in Statistics from University of Madras. He is currently working as Associate Professor, Department of Statistics, Pondicherry University. He has more than 25 years of experience both in Teaching and Research. To his academic credit he has received U.S.Nair Young Statistician Award from Indian Society of Probability and Statistics and also the International Young Statistician Award from the International Statistical Institute, The Netherlands. His research interests are Estimation of Variance Components, Missing Data Analysis, Sampling Theory, Incomplete Block Designs, Control Charts and Process Capability Analysis. He has published more than 75 research papers in reputed International and National Journals. He has also participated in many conferences and workshops in India as well as abroad and presented research papers; delivered invited talks and special addresses. $\mathrm{He}$ has organized many International and National Conferences and also acted as an organizing committee member in many conferences. 